Alter

Revue de phénoménologie

$20 \mid 2012$

Eros

\title{
L'Erôs et la désublimation du désir
}

\section{Renaud Barbaras}

\section{OpenEdition}

\section{Journals}

Édition électronique

URL : http://journals.openedition.org/alter/997

DOI : 10.4000/alter.997

ISSN : 2558-7927

\section{Éditeur :}

Association ALTER, Archives Husserl (CNRS-UMR 8547)

\section{Édition imprimée}

Date de publication : 1 décembre 2012

Pagination : 13-26

ISBN : 978-2-95-223748-2

ISSN : 1249-8947

\section{Référence électronique}

Renaud Barbaras, "L'Erôs et la désublimation du désir 》, Alter [En ligne], 20 | 2012, mis en ligne le 01 juillet 2019, consulté le 28 juillet 2019. URL : http://journals.openedition.org/alter/997 ; DOI : 10.4000/ alter.997 


\section{L'EROS ET LA DÉSUBLIMATION DU DÉSIR}

Publié une première fois sous ce titre dans La vie lacunaire, Paris, Vrin, 2011.

Renaud Barbaras

L'Erôs est traditionnellement considéré comme relevant du registre de l'affectivité, lui-même opposé à celui de la connaissance en son sens le plus large. Cela signifie que si l'Erôs enveloppe bien un rapport à un autre, néanmoins, il ne dévoile rien, ne fait rien connaître par lui-même. Il suppose au contraire que son objet soit déjà donné afin de pouvoir se rapporter à lui ; il appelle un voir préalable comme la condition de son désir. C'est ce que Husserl luimême résumait à travers l'affirmation du primat des actes objectivants sur les actes non-objectivants, ce qui signifie que le désir, par exemple, loin de constituer ou de dévoiler son propre objet, suppose que celui-ci lui soit préalablement donné dans une représentation : il doit d'abord être objet pour être objet du désir. Une fois ces deux sphères soigneusement distinguées, comme deux modes de rapports absolument irréductibles l'un à l'autre et d'une dignité très inégale quant à leur aptitude à découvrir quelque chose, il devient particulièrement délicat de penser une articulation possible entre les deux. Celle-ci ne peut être ressaisie au plan du rapport lui-même mais seulement de l'énergie qui y est engagée : l'énergie érotique serait susceptible de substituer au but proprement sexuel qu'elle se donne d'abord un but d'un autre ordre. Ainsi, l'activité de connaissance serait encore une manifestation, pour ainsi dire détournée, de l'énergie qui est à l'œuvre dans l'Erôs, bref une sublimation, terme dans l'usage duquel il faut entendre les connotations à la fois chimiques et esthétiques. C'est évidemment à Freud que l'on doit cette hypothèse selon laquelle « la pulsion sexuelle met à la disposition du 
travail culturel des quantités de forces extraordinairement grandes et cela par suite de cette particularité, spécialement marquée chez elle, de pouvoir déplacer son but sans perdre pour l'essentiel de son intensité. On nomme cette capacité d'échanger le but sexuel originaire contre un autre but, qui n'est plus sexuel, mais qui lui est psychiquement apparenté, capacité de sublimation $»^{1}$.

Cette hypothèse soulève plus de problèmes qu'elle n'en résout. Comment comprendre que l'énergie érotique puisse se détourner de son but sexuel initial ? Ne faut-il pas admettre, ce que Freud luimême fera, une limitation ou une insatisfaction au niveau même de la pulsion sexuelle, qui expliquerait qu'elle puisse se détourner par ellemême de son but? Surtout, d'autre part, afin qu'une telle conversion soit possible, il est nécessaire de supposer une équivalence autre que strictement énergétique entre ces deux modes de relation à l'objet : le désir ne peut se muer en connaissance que dans la mesure où il n'est pas étranger à la connaissance, où celle-ci n'est pas en rupture avec le désir. Par les difficultés qu'elle soulève, la théorie de la sublimation invite à dépasser le clivage qu'elle présuppose entre l'Erôs et la connaissance, entre le désir et le voir, au profit d'une dimension plus originaire, à la faveur de laquelle pourrait s'effectuer justement le passage de l'un à l'autre, la conversion d'une énergie dans une autre. Autrement dit, si le désir peut se faire connaissance, n'est-ce pas parce qu'il est déjà connaissance, c'est-à-dire exerce par lui-même une fonction dévoilante, que la connaissance proprement dite ne ferait alors que reprendre à son compte et prolonger ? Plus profond que le partage entre l'Erôs et la connaissance, il y aurait un Désir originairement dévoilant qui serait non seulement la raison mais l'élément même de ce passage mystérieux que Freud nomme sublimation. Mais, dire qu'il y a un désir originairement dévoilant, c'est reconnaître que dans tout dévoilement, c'est-à-dire dans la phénoménalité comme telle, un désir est à l'œuvre. C'est donc sur le terrain phénoménologique, celui où peut être posée la question de la phénoménalité des phénomènes, qu'une telle thèse doit être mise à l'épreuve. $C^{\prime}$ est ce à quoi nous nous attacherons maintenant. Cependant, dans une telle perspective, ce qui allait jusqu'alors de soi et constituait un point de départ, à savoir l'Erôs comme désir de l'autre, fait soudain problème et devient énigmatique. Si l'on découvre un désir au cœur de l'apparaître, quelle peut bien être la spécificité de ce que nous nommons proprement désir, pour autant qu'il semble, quant à lui, étranger au voir et au connaître ? Quelle peut donc être la place de

${ }^{1}$ Die „kulturelle“ Sexualmoral und die moderne Nervosität, 1908, G.W., VII, 150. 
l'Erôs dans une phénoménologie qui reconnaît un désir originaire au cœur de toute phénoménalisation?

Il suffit de partir de la thématisation husserlienne de l'intuition et, plus particulièrement, de cette intuition donatrice originaire qu'est la perception, pour découvrir que cette théorie de l'intuition est comme condamnée à se déborder elle-même au profit d'une dimension encore plus profonde. On le sait, chez Husserl, l'intuition signifie toujours le remplissement d'une visée à vide (signitive) : elle est donation en personne (Leibhaft) de cela qui, jusqu'alors, n'était visé qu'à vide, c'est-à-dire donné in absentia. Dans le cas de la perception qui, en tant qu'intuition originaire, délivre l'Être même (Lévinas), cette présence en personne repose sur une donation par esquisses. Comme l'écrit Husserl, "En vertu d'une nécessité eidétique, une conscience empirique de la même chose perçue sous "toutes ses faces", et qui se confirme continuellement en elle-même de manière à ne former qu'une unique perception, comporte un système complexe formé par un divers ininterrompu d'apparences et d'esquisses; dans ce divers viennent s'esquisser eux-mêmes (sich abschatten), à travers une continuité déterminée, tous les moments de l'objet qui s'offrent dans la perception avec le caractère de se donner soi-même corporellement $»^{2}$. Autrement dit, sous l'autorité des esquisses actuelles et du cours déjà écoulé, une unité est anticipée, c'est-à-dire visée, unité qui va être confirmée ou infirmée par de nouvelles esquisses. L'objet comme tel est le corrélat de ce cours concordant d'esquisses, le pôle ultime d'une anticipation confirmée, ce qui revient à dire que c'est sur la base d'un dépassement des esquisses actuelles, d'une continuation de l'expérience, que cet objet peut se constituer. Cependant, une difficulté majeure surgit aussitôt. Si, à chaque instant, les esquisses actuelles peuvent être dépassées et l'expérience se poursuivre, c'est dans la mesure où cette possibilité m'est d'emblée garantie, où j'ai la certitude de la continuabilité de l'expérience. Or, cette garantie ne peut évidemment pas reposer sur l'objet puisque celui-ci est au contraire ce qui est constitué par le cours convergent de l'expérience. Il faut donc admettre que, afin que le cours des esquisses se déroule, je dois disposer du cadre préalable au sein duquel cette expérience peut être poursuivie, du champ dans lequel des objets peuvent être constitués : la continuation de l'expérience grâce à laquelle me sont donnés des objets suppose que me soit d'abord donnée la continuabilité de cette expérience, sur un mode qui ne saurait être lui-même objectif. Comme le résume Merleau-Ponty, ce n'est pas parce que je

${ }^{2}$ Ideen.I, trad. Ricoeur, Paris, Gallimard, 1950, p. 132. 
peux poursuivre les esquisses de manière convergente que je sais qu'une chose est là ; c'est au contraire parce que la chose est là que je sais que je vais pouvoir poursuivre le cours des esquisses. Il faudrait seulement ajouter que ce qui est déjà là comme garantie de la continuabilité de l'expérience n'est évidemment pas une chose et que le cours des esquisses peut être tel que mes anticipations soient infirmées. Il pourrait même arriver qu'elles le soient toujours, au point que je n'aurais jamais affaire à des choses mais toujours et seulement à un pur chaos. Néanmoins, contrairement à ce que dit Husserl, une telle situation, fruit de la variation eidétique, ne signifierait en aucun cas la disparition du monde : comme on va le voir, elle révèle au contraire l'essence du monde, précisément comme cela qui est donné d'emblée comme cadre ou fond permettant le développement même de l'expérience et donc comme cela qui est ultimement figuré par les esquisses, même si celles-ci ne parviennent pas à devenir esquisses d'objets. Comme l'écrit on ne peut plus clairement Patočka : "Que j'aie toujours à nouveau, où que je me trouve, la possibilité de réaliser la même continuation, cela n'est pas simplement anticipé, mais donné, sous la forme, non pas d'une simple intention, mais d'une présence indépendante du remplissement contingent ou de la simple anticipation vide $»^{3}$. L'important ici est que la possibilité de continuer l'expérience ne soit pas possédée sous la forme d'une potentialité de la conscience, comme c'était le cas chez Husserl, mais donnée sous la forme d'une présence plus originaire que celle de l'objet.

La conséquence, décisive pour notre propos, de cette analyse, est la disjonction entre la donation en chair - c'est-à-dire l'originarité - et l'intuition. Dire que le cadre de la continuabilité de l'expérience doit déjà être là pour qu'un objet soit constitué, ou encore pour qu'une intuition perceptive soit possible, c'est reconnaître qu'il y a un plan qui est plus profond que celui de l'intuition et qui en constitue la garantie. Au fond, tout se passe comme si la visée à vide, sur laquelle repose la poursuite de l'expérience, possédait un mode de remplissement propre, comme si cette visée à vide avait pour corrélat une certaine présence qui, pour être vide d'objet, n'en était pas pour autant non-être. Telle est sans doute la raison pour laquelle Patočka insiste sur le fait que « le vide n'est en aucun cas une non-donation,

\footnotetext{
${ }^{3}$ Papiers Phénoménologiques, trad. E. Abrams, Grenoble, J. Millon, 1995, p. 178. Noté désormais PP. Cf aussi p. 184 : «Il est vrai que ce qui est absent ne peut pas être présent; mais le fait que toute présence fait partie d'une seule et unique présence englobante peut, lui, être présent ; cette structure, portant sur la totalité, peut être immédiatement présente ».
} 
mais un mode de donnée »4. Toute la question est celle de savoir ce qui donne ce vide pour autant que cela ne saurait être une intuition. Quoi qu'il en soit, la condition de la perception elle-même est bien la disjonction de cela que Husserl identifiait, à savoir la donation en chair et la présence intuitive : celle-ci n'est désormais pensable, en tant qu'elle enveloppe un cours d'esquisses et un procès d'anticipation, que sur fond d'une présence pré-donnée, d'une archi-originarité qui ne peut être intuitive puisqu'elle est au contraire cela que toute intuition présuppose et vient déterminer. Cette disjonction est patente dans ces exemples que donne Patočka : "Que la face arrière de la table que voici ne soit pas présente en personne, cela ne signifie pas que ne soit pas présent en personne le fait que la table en tant qu'objet physique a nécessairement une face arrière. La même chose vaut pour l'appréhension de l'espace - le fait que l'espace soit toujours donné uniquement d'un point de vue ne signifie pas que ne soit pas donné sur le mode de soi-même le fait que chacun de ces points de vue avec la fraction d'espace qu'il saisit fait partie du seul et unique espace omni-englobant $»^{5}$.

On l'aura compris, cette présence originaire et non intuitive qui fonctionne comme sol pour la perception n'est autre que le monde. Celui-ci est en effet le cadre ou la scène préalable au sein desquels toute apparition peut avoir lieu, l'apparaissant ultime qui est au cœur de toute apparition et, par conséquent, co-apparaît en toute apparition comme sa condition ou son élément propre. Donné originairement et pour ainsi dire plus profondément que toute apparition en son sein, il n'est cependant jamais donné intuitivement, comme un contenu ou un étant, car il fonctionne plutôt comme forme pour toute apparition. Il est à la fois et nécessairement du côté du donné et du côté de sa condition, comme le cadre présupposé par tout donné ; il est cet archi-donné qui est au cœur de toute donation. Le monde est ce fond qui est aussi forme et c'est pourquoi Patočka le décrit comme un plan d'apparition qui est en même temps instance d'apparition, comme un datum non pas empirique mais apriorique, comme forme pour ses propres contenus ${ }^{6}$. Pour le dire autrement, ce monde n'est évidemment pas un étant puisqu'il est la condition ultime et pré-donnée de ceux qui apparaissent en son sein : en ce sens il est non-étant, il est une "non-chose $»^{7}$. Mais, précisément dans la mesure où il est une non-chose, il ne peut non

\footnotetext{
4 PP, p. 176.

${ }^{5}$ PP, p. 178.

${ }^{6} \mathrm{PP}$, pp. 122, 181, 214.

7 PP, p. 221.
} 
plus être autre chose, autre chose que les étants eux-mêmes, car cela serait retomber encore au plan de l'étant et de sa positivité ; ce serait présupposer un autre monde comme nouveau cadre de cette nouvelle étantité. Dire qu'il excède les choses, c'est dire qu'il n'est rien d'autre et dire qu'il n'est rien d'autre c'est au fond souligner qu'il n'est autre qu'en n'étant rien (d'étant) - rien de plus que les étants qu'il totalise. Ainsi, il ne peut être non-chose qu'à la condition d'être tout autant non-autre, non-aliud ${ }^{8}$. Ce rien d'étant, ce non-intuitif originairement donné comme sous-jacent à toute apparition existe nécessairement sur le mode d'une transcendance pure sans contenu propre, d'un excès qui n'implique aucune altérité, bref d'une différence nue, qui est « différence des identiques ${ }^{9}$. C'est comme si l'ensemble des étants s'était originairement et inchoativement différencié de luimême, en une sorte de degré zéro de la différence, pour se faire sa propre condition, s'envelopper lui-même, c'est-à-dire se muer en totalité omni-englobante et ouverte.

Cette dimension originaire du monde exige bien sûr un autre mode de donation que celui de l'intuition ; la négativité qui le caractérise est incompatible avec toute forme d'intuitivité. Pourtant, puisqu'il y a un monde pour nous (et il y a un monde pour nous dès qu'il y a des choses pour nous) il faut bien que nous soyons capables de l'effectuer, de le faire paraître selon son être propre qui est un nonétantité. En ce sens, la mise au jour du monde au cœur de l'apparaître ouvre la voie d'une détermination neuve et plus radicale du sujet luimême. Ce sujet ne peut plus être caractérisé par le voir, son rapport au monde ne peut être de mise en présence au sens d'une coïncidence ; s'il est habité par une visée, celle-ci est désormais d'une nature telle qu'elle ne saurait être remplie. À dire vrai, pour autant que le monde est bien une transcendance sans contenu, un pur excès ou un négativité qui n'est pas l'envers d'une altérité, il ne peut être donné que comme le pôle d'une avancée ou d'une approche, possédé qu'en étant parcouru, présent qu'en étant inlassablement rempli. Le monde est cela vers quoi nous tendons sans jamais parvenir à le rejoindre, cela que nous rendons présent sans jamais parvenir à l'épuiser. À un être qui excède indéfiniment tout présence intuitive, puisqu'il est la totalité ouverte des apparitions, ne peut correspondre qu'un sujet qui est aspiration : bref, le monde ne peut être donné comme tel que sur le mode du désir. Le monde est ce qui est désiré, non pas au sens d'un déjà là qui, pour ainsi dire après-coup, ferait

\footnotetext{
${ }^{8}$ PP, p. 122.

${ }_{9}^{9}$ Merleau-Ponty, Le visible et l'invisible, Paris, Gallimard, 1964, p. 316.
} 
l'objet d'un désir, mais en tant que c'est dans le désir et seulement en lui que son être propre nous est délivré. Parce qu'il est non-chose, pur excès sur l'étant, le monde n'est pas ce que nous voyons ou connaissons mais ce vers quoi nous tendons, à quoi nous aspirons, ou plutôt $c^{\prime}$ est dans cette aspiration qu'il est saisi conformément à son être, c'est dans le caractère inextinguible du désir que se préserve son irréductible trancendance. Ainsi, la découverte du monde nous a conduit à approfondir le sens d'être du sujet: en traversant la présence perceptive au profit du monde qui la commande, nous avons dépassé le sujet intuitif au profit du désir qui le fait être. Mais il faut comprendre le sens de ce désir à la lumière du monde plutôt que sur la base des vécus que nous lui annexons spontanément : il est la condition d'apparaître du monde comme tel, son transcendantal propre et, à ce titre, une aspiration sans satiété possible, une avancée sans repos.

En repensant le désir dans un cadre phénoménologique, en suspendant sa référence spontanée à la sphère de l'affectivité et des besoins, nous n'en forçons pas la signification mais en révélons au contraire la teneur de sens authentique. Nous avons découvert que le monde comme tel ne se donne qu'au désir, mais il faut maintenant ajouter qu'il n'y a de désir que comme désir d'un monde, que cette aspiration singulière qui est à l'œuvre dans la donation d'une originarité non-intuitive délivre en vérité l'essence du désir. Si le désir est la condition du monde, celui-ci est la vérité du désir. En effet, dans le cadre de la donation du monde, ce désir par lequel nous désignons l'intentionnalité la plus originaire peut être caractérisé par une double exclusion, mettant ainsi fin à une double confusion. Nous l'avons dit, le monde est non-aliud, il n'est pas autre, pas autre que les étants qui paraissent en son sein. C'est pourquoi - il est temps de le préciser - la donation du monde ne se distingue pas, sinon en droit, de la perception des étants au sein du monde. Le monde est ce rien qui ne se donne que sous la forme de son propre remplissement, cette archiprésence constamment convertie en intuition. Autant dire que la visée du monde non seulement exige un remplissement mais se confond avec lui, pourvu que l'on comprenne ce remplissement sur le mode dynamique. La donation du monde n'est autre que l'œuvre du remplissement intuitif, c'est-à-dire de la perception; en ce sens, non seulement l'excès du monde ne signifie rien d'autre que celui de la visée même sur sa réalisation mais cet excès ne se découvre que dans et par cette réalisation. Le monde est cela qui se donne en chaque réalisation comme transcendant à chaque fois la figure finie (les apparitions) sous laquelle il se réalise. Pour cette raison, c'est bien de désir qu'il s'agit dans la donation du monde, pour autant que le désir enveloppe 
sa propre réalisation, qu'un désir qui renoncerait d'emblée à la satisfaction ou ne pourrait d'aucune façon y prétendre ne serait pas un désir. À l'inverse, un désir qui serait rapport à un tout autre, comme chez Levinas, "désir sans satisfaction » qui "entend l'éloignement, l'altérité et l'extériorité de l'Autre », désir « qui n'aspire pas au retour car il est désir $\mathrm{d}^{\prime}$ un pays où ne naquîmes point ${ }^{10}$, un tel désir donc, que Lévinas nomme "désir métaphysique », n'est en aucun cas un désir. Une tension sans satisfaction ne peut être celle du désir et c'est pourquoi, parce qu'il se réalise comme séparation avec le désiré et épreuve de la séparation, parce que non seulement il exclut la satisfaction mais s'accomplit comme ce renoncement même, le prétendu désir métaphysique est étranger au désir : il est en vérité plutôt responsabilité ou sainteté. Seule relève donc du désir la visée du monde en tant qu'elle comporte un horizon de satisfaction et permet par conséquent une avancée. La co-appartenance de l'originaire et de l'intuitif, du monde et de ses apparitions, telle que la distance du monde ne se donne que dans ce qui la comble, est exactement ce qui garantit l'effectivité, l'horizon de satisfaction sans lesquels on ne peut parler de désir.

Cependant, le remplissement ne signifie pas la plénitude et la satisfaction n'est pas synonyme de satiété. Si l'excès du monde sur ses apparitions est inassignable (à un autre) et n'est donc pas l'envers d'une altérité, il est néanmoins irréductible. Dire que le monde n'est rien d'étant, c'est reconnaître que l'intentionnalité qui le soutient ne peut être comblée, que la visée à vide excède toujours son remplissement, le monde n'étant au fond rien d'autre que l'excès renouvelé de ce vide sur ce qui vient le remplir. En tant que non-chose ou transcendance pure, le vide du monde est un vide incomblable. Or, c'est précisément ce qui distingue le désir du manque et, en particulier, de ce manque déterminé et réitéré qu'est le besoin. Le propre du manque est d'être manque de quelque chose, il dessine toujours en creux les contours de ce qui le remplira ; c'est aussi la raison pour laquelle la possession de l'objet du besoin signifie l'extinction de ce besoin. On pourrait donc dire que penser le désir comme manque et le rabattre par conséquent sur le besoin, c'est situer sur le plan ontique ce qui excède ce plan, c'est projeter le monde sur le plan de l'objet et confondre par là même le véritable objet du désir avec une chose du monde. Mais c'est là en manquer évidemment l'essence pour autant que, s'il appelle bien une satisfaction, le désir est néanmoins tel que ce qui le satisfait ne le comble pas, que ce qui

10 Totalité et infini, La Haye, Martinus Nijhoff, 1961, pp. 3,4. 
l'apaise en même temps l'exacerbe. Le désir ne manque de rien car rien ne le comble vraiment : sa portée excède celle du manque, c'està-dire de l'objet, quel qu'il soit. Ainsi, affirmer que le désir n'est pas le besoin puisque rien ne l'éteint, pas même ce qui le satisfait, c'est dire qu'il ne désire rien de déterminé et $c^{\prime}$ est par conséquent reconnaître qu'il n'y a de désir que portant sur cela qui, excédant l'ordre des étants, n'est pourtant pas autre, à savoir précisément le monde luimême. Identifier l'essence du désir dans sa différence avec le besoin c'est le rapporter au monde comme à son unique corrélat possible. Comme nous l'avons annoncé, en remontant du monde à sa condition véritable, c'est bien à l'essence du désir que nous accédons, en sa différence avec ce avec quoi il demeure toujours possible de le confondre. Si le désir se distingue de la responsabilité levinassienne comme le Même se distingue de l'Autre, il se distingue tout autant $\mathrm{du}$ besoin comme le monde se distingue des étants qui apparaissent en son sein. De sorte que, à la condition de transcender l'acception commune des termes pour y voir une manière de nommer une modalité singulière de rapport, on pourrait dire que si le rapport à l'autre en son altérité est responsabilité, et le rapport au monde comme tel désir, l'intuition comme remplissement de la visée à vide, ou encore coïncidence, plénitude, est besoin. La responsabilité se rapporte sur le mode de la séparation, le désir sur le mode de la transcendance et le besoin sur le mode de la possession. Bref, l'autre est à la responsabilité ce que le monde est au désir et l'étant intramondain au besoin.

Contre toute attente, c'est dans un contexte théologique que nous trouvons les formulations les plus justes quant au concept de désir que nous tentons de construire ici. Il s'agit de la description de l'épectase par Grégoire de Nysse dans La vie de Moïse. Le mouvement de l'âme vers Dieu est qualifié par un désir qui est décrit dans des termes où se révèle sa véritable essence, de telle sorte qu'il suffirait de mettre le monde à la place de Dieu, si l'on peut dire, pour y voir une description phénoménologique tout à fait satisfaisante de ce dont il y va dans le désir. Le désir, écrit Grégoire de Nysse, que l'âme a de ne pas renoncer "aux sommets qui sont au-dessus d'elle, lui communique un mouvement ascensionnel qui n'a pas de cesse, où elle trouve toujours dans ce qu'elle a réalisé, un nouvel élan pour voler plus haut. Seule en effet l'activité spirituelle a cette propriété de nourrir sa force en la dépensant et de ne pas perdre mais d'augmenter sa vigueur par l'exercice ${ }^{11}$. Telle est bien la loi du désir que

${ }^{11}$ La vie de Moïse, trad. J. Daniélou, Paris, Cerf, 2007, p. 263. 
de s'augmenter en se satisfaisant et donc de se renouveler en se dépensant, mais c'est là en vérité la loi même du monde, qui recule devant celui qui le fait paraître et appelle donc le dépassement de toute apparition finie, dès lors que celle-ci dessine en le niant l'infini qui l'excède. Il n'y a d'épreuve du monde en son infinité que sous la forme de cette insatisfaction qui renaît en toute satisfaction, de cette force qui s'augmente de se dépenser. Comme le dit magnifiquement Grégoire de Nysse, «la voix divine accorde ce qui est demandé par le fait même qu'elle le refuse [...]. En effet la munificence de Dieu lui accorde l'accomplissement de son désir; mais en même temps elle ne lui promet pas le repos ou la satiété $»^{12}$. Cette formulation vaut encore parfaitement pour le monde, dont on pourrait dire qu'il est accordé en son être de monde dans la mesure où il se refuse à l'intuition. C'est précisément ce refus ou cette traversée de l'intuition que nomme le désir en tant que, pour lui, l'apaisement n'est jamais repos, la satisfaction jamais satiété. Telle est la loi conjointe du désir et du monde : parce qu'aucun étant n'épuise le monde, la satisfaction ne peut se faire satiété. Or, enfin un tel désir ne doit en aucun cas être situé en opposition au connaitre : il n'est pas l'autre de la vision mais une autre vision, précisément celle qui, selon nous, convient à l'être du monde. C'est pourquoi Grégoire de Nysse peut conclure, dans le contexte qui est le sien : "C'est là réellement voir Dieu que de ne jamais trouver de satiété à ce désir $»^{13}$. Si on donne au voir le sens originaire d'un dévoilement, c'est-à-dire d'un accès à l'être de cela qui est « vu », il faut affirmer qu'il y a deux sens du voir, et tout notre propos a visé au fond à les distinguer. Il y a le voir au sens étroit, compris comme intuition : c'est le voir husserlien, le voir des yeux et de l'esprit. Il y a le voir comme accès à cela qui excède l'intuition et la commande, comme ouverture du monde ; c'est le voir d'un invisible qui, à ce titre, implique une quête : ce voir est désir. Cela signifie que, seul, il peut viser un rien d'étant qui n'est pourtant rien d'autre que l'étant, car en son insatisfaction se donne ce qui se refuse à l'intuition. L'insatisfaction du désir se rapporte à un vide qui n'est autre que l'appel de son remplissement: en cela, le désir est bien l'ouverture même du monde.

Nous pouvons maintenant revenir à notre point de départ. En portant la question sur le terrain de la phénoménologie et plus particulièrement de l'analyse de l'être du monde, nous avons accédé à un sens originaire du désir qui est étranger au partage de l'affectivité et 
de la connaissance, de la tendance et de la vision, car il est plus profond qu'eux. À l'être du monde correspond un mode de dévoilement qui n'est pas intuition mais aspiration, avancée plutôt que contemplation, dépossession plutôt que possession. En nous interrogeant sur la condition subjective du monde, nous avons découvert une vision qui est désir, un dévoilement qui prend la forme de l'approche. Seulement, dire qu'il y a une vision originaire, c'est-à-dire un dévoilement du monde qui est en son fond désir, c'est aussi reconnaître que le désir, dont le passage par le monde nous a révélé l'essence, est toujours aussi vision, dévoilement de son objet. Désirer c'est au fond faire paraître l'objet en tendant vers lui, le faire être en tentant de se l'approprier et se découvrir soi-même dans cette avancée. C'est pourquoi il faut conclure, à l'encontre de ce que le premier Husserl avançait, qu'aucun acte objectivant n'est requis pour que soit donné un objet susceptible d'être désiré : le désir constitue au contraire son objet, le fait être par son désir même. Surtout, d'autre part, le problème que soulevait d'idée de sublimation se trouve par là même résolu. En effet, c'est seulement parce que l'on postule leur hétérogénéité foncière que l'on en vient à se demander comment du désir peut se faire connaissance, comment une pulsion peut devenir vision et que l'on est alors conduit à faire l'hypothèse d'une même énergie qui se donne un autre but que son but sexuel initial. En vérité, l'hypothèse de la sublimation disparaît avec le présupposé qui la commande. Le désir peut se faire connaissance tout simplement parce qu'il est toujours déjà un voir et n'a jamais été une simple énergie pulsionnelle aveugle. Comme nous l'avons dit en commençant, on se demande comment cette conversion peut avoir lieu dès lors qu'elle concerne des rapports à l'objet qui sont foncièrement hétérogènes. Mais c'est précisément cette difficulté que notre théorie du désir permet de surmonter. La possibilité de la sublimation est fondée sur l'essence dévoilante du désir : parce que, dès le niveau pulsionnel, c'est déjà de dévoilement qu'il s'agit, parce que nous avons donc affaire de part et d'autre au même mode de rapport au monde, le passage de l'un à l'autre est possible. La possibilité de cette conversion manifeste qu'il n'y a pas deux sphères mais une seule, qu'il n'y a pas de désir qui ne soit connaissance au sens du dévoilement, pas de tendance qui ne soit déjà vision. Ainsi, contre toute attente, une analyse critique du concept de sublimation vient converger avec les résultats les plus avancés de la phénoménologie de la perception. Autant dire alors que la sublimation est un concept auquel il faut renoncer car soit elle demeure incompréhensible, si l'on demeure dans le cadre conceptuel au sein duquel elle a été formulée, soit elle a 
toujours déjà eu lieu. Au fond, nous pourrions résumer ce que nous avançons, dans les termes de la conceptualité dualiste que nous sommes sur le point d'abandonner, en disant que le désir est sa propre sublimation pour autant que la poussée qui le caractérise est déjà vision, dévoilement d'un monde, ou encore que la sublimation est au cœur du désir puisque son être est à la charnière de l'avancée et de la phénoménalisation et, en vérité, plus profond que cette distinction même.

Il reste néanmoins une difficulté, qui n'est pas des moindres. Même si, par la médiation de l'analyse de l'être du monde, nous sommes parvenus à mettre en évidence une essence du désir, il n'en reste pas moins que nous avons aussi affaire à un désir proprement érotique qui nous porte vers l'autre, qu'il y a une évidence phénoménologique de l'Erôs. Comment donc situer l'Erôs dans cette phénoménologie du désir sans réinstaurer la coupure entre un désir qui serait nécessairement et exclusivement érotique et un rapport au monde étranger au désir? Comment faire place à l'Erôs sans lui sacrifier l'essence du désir? Il n'y a qu'une seule réponse possible : l'Erôs doit être compris comme une désublimation du désir. Bien entendu, il faut tout de suite ajouter, sauf à tomber dans l'incohérence, que ce concept de désublimation est, en dépit de sa forme négative, un concept premier et original. Il ne renvoie pas à une sublimation préalable dont il serait la négation, pas plus que l'inconscient freudien ne peut être caractérisé comme négation de la conscience. Il n'y a pas de sublimation du désir, pour autant que celui-ci est toujours déjà dévoilant, mais il y a une désublimation du désir dans l'Erôs. Nous entendons par là un procès qui est inverse de celui que Freud décrit - et $c^{\prime}$ est pourquoi nous retenons ce terme - c'est-à-dire quelque chose comme un reflux, une cristallisation et une singularisation ${ }^{14}$. Reflux $\mathrm{du}$ désir en sa dimension dévoilante vers une pure aspiration (qui n'est pas une aspiration pure), cristallisation de la forme totale du monde dans un étant intra-mondain, enfin, singularisation de sa généralité constitutive sous la forme de cet autre. Tout se passe comme si, dans l'amour, l'excès constitutif du monde refluait pour venir prendre fugitivement place en lui, comme si son invisibilité principielle prenait soudain figure visible. En d'autres termes, en une sorte d'aberration phénoménologique, tout se passe comme si la condition même de l'apparaître était sur le point d'apparaître. C'est sans

\footnotetext{
${ }^{14}$ Encore faut-il souligner que ce que nous nommons désublimation n'est finalement pas très éloigné de ce que Lacan entend par sublimation. Il la définit en effet ainsi : « Elle élève un objet à la dignité de chose » (Séminaire VII, p. 133).
} 
doute ce que voulaient dire tous ceux qui ont souligné le fait qu'aimer l'autre, c'est toujours être fasciné par un monde, c'est aimer son monde et, pourrions-nous ajouter, à travers son monde, accéder au monde même. Dans l'amour, se donne soudain sur le mode intuitif ce qui excède l'ordre de l'intuitionnable. Mais encore faut-il s'entendre. Cela ne signifie pas que l'amour est de l'ordre de l'intuition, comme si en l'autre devenait soudain visible l'invisible du monde. Cela signifie plutôt que nous avons affaire, dans l'amour, à une expérience absolument singulière, celle d'une percée du monde en l'autre ou d'une traversée de l'autre vers le monde. Il ne faut donc pas dire qu'en l'autre l'invisibilité du monde se visibilise mais plutôt que l'amour nomme une épreuve qui n'a pas d'autre nom, l'épreuve de cette percée, de cette quasi-présence du monde en l'autre. Aimer c'est pressentir en l'autre le visage du monde et tendre vers lui comme vers celui qui m'en délivrera la clé, comme s'il concentrait soudain en lui la puissance phénoménalisante du monde. Cette expérience singulière est donc aussi une expérience limite, au sens strict, c'est-à-dire au sens où elle se situe à la limite, ou plutôt est la saisie d'une limite : précisément de celle, à la fois irréductible et disparaissante, qui sépare le monde de ses manifestations mondaines. Dans l'amour, le monde semble refluer sur lui-même et l'éclat que l'autre y acquiert tient finalement au fait qu'il semble se tenir à la lisière du monde, au point de ce pur excès qu'est le monde. Dans l'amour, l'illimitation du monde semble se rassembler en son sein, se concentrer à sa propre limite, là où peut surgir un être fini, qui vient comme témoigner de sa profondeur.

Dans la Huitième Elégie de Duino, Rilke présente précisément l'expérience amoureuse comme une expérience limite, qui contrevient à notre condition. Celle-ci est décrite dans une opposition à ce qu'il nomme la créature, c'est-à-dire l'animal, opposition qui est celle de la vue animale et du regard humain : " $D^{\prime}$ 'une pleine vue la créature voit/ l'Ouvert. Seuls nos yeux sont/ comme à rebours, posés tout autour d'elle/ainsi que pièges, cernant sa libre issue $»^{15}$. Or, l'expérience amoureuse est celle du brouillage de ce partage : « Les amants, si ce n'était l'autre, qui/masque la vue, en sont proches et s'étonnent.../Comme par surprise cela leur est ouvert/ derrière l'autre... Mais nul/ne franchit l'autre et pour l'amant c'est à nouveau le/ monde $»^{16}$. Bien entendu, à la distinction entre ce que Rilke nomme l'ouvert et le monde il faut substituer celle entre ce que nous avons

\footnotetext{
${ }_{15}$ Trad. R. Munier, dans Parcours Oblique, Paris, La Différence, 1979, p. 81.

${ }^{16}$ Ibid., p. 81.
} 
nommé le monde comme tel et la réalité intra-mondaine. Mais quant à l'amour, il nous semble que c'est bien de cette épreuve qu'il s'agit : d'une percée du monde (ou de l'ouvert) en ou à travers l'autre, d'une prise du monde même dans un visage, celui d'un autre au sein de ce monde. C'est cette épreuve que Roger Munier décrit en des termes particulièrement heureux : «A la faveur du mouvement qui les porte, les amants aussi vont aux confins. Derrière l'autre et son pouvoir d'arrachement et d'appel, l'au-delà lumineux comme par surprise leur est ouvert. Ils en sont proches, dans leur élan et s'étonnent. L'amour aussi déprend du monde [il faut entendre aussi du règne de l'objet], mais étrangement y ramène. Car il ne va qu'à l'autre, et l'autre est du monde encore, contrariant par là-même le mouvement qu'il suscite et qui s'ébauche seulement ${ }^{17}$. L'amour est cette modalité singulière du désir, modalité désublimée, par laquelle soudain il se porte vers un étant du monde comme l'attestation à la lisière du visible du pur excès du monde et de sa totalité sans limites. En vérité, si elle correspond à ce dont nous faisons l'expérience, cette description est pour ainsi dire appelée par les conditions mêmes du problème : puisque le désir est toujours donation du monde et puisqu'il y a un désir érotique de l'autre, ce désir ne peut que signifier l'accès au monde en l'autre, au sens où le monde paraît ou se figure en lui. L'amour est cette épreuve sans nom d'une rencontre fugitive entre l'Appaissant originaire et une certaine apparition, $d^{\prime}$ 'un brouillage de la frontière entre la totalité et cela qu'elle totalise, d'un repli ou d'une involution du monde sur lui-même. Enfin, il peut se produire que l'Erôs continue pour ainsi dire sur sa lancée pour s'épanouir comme désir au sens où nous l'avons défini, c'est-à-dire pour se faire quête du monde lui-même, quelle que soit la modalité de cette quête. C'est alors que l'on peut parler d'une sublimation de l'Erôs ; mais cette sublimation est la négation d'une désublimation qui est plus originaire qu'elle et qui la conditionne : ici, la formule freudienne se trouve purement et simplement renversée. Or, cette sublimation seconde est toujours possible car elle ne fait que retrouver le mouvement originaire du désir que l'Erôs avait nié à sa façon, car elle repasse sur le pointillé d'une phénoménalisation originaire que l'on avait cru pouvoir suspendre dans l'amour pour se l'approprier en la fixant au sein du monde.

17 Ibid., p. 94. 